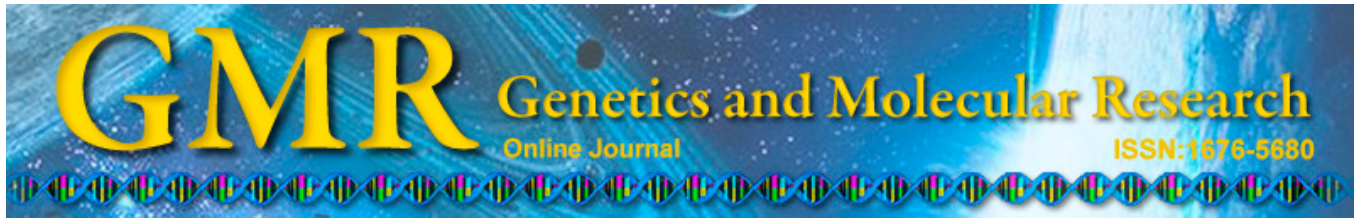

\title{
In vivo mechanism study of NGAL in rat renal ischemia-reperfusion injury
}

\author{
X.J. Zang', S.X. An ${ }^{2}$, Z. Feng ${ }^{1}$, Y.P. Xia ${ }^{1}$, Y. Song' ${ }^{1}$ and Q. Yu ${ }^{2}$ \\ ${ }^{1}$ Department of Nephrology, \\ Songjiang Branch of the First Affiliated People's Hospital, \\ Shanghai Jiaotong University, Shanghai, China \\ ${ }^{2}$ Department of Nephrology, The First Affiliated People's Hospital, \\ Shanghai Jiaotong University, Shanghai, China \\ Corresponding author: X.J. Zang \\ E-mail: zangxiujuan@163.com
}

Genet. Mol. Res. 13 (4): 8740-8748 (2014)

Received October 7, 2013

Accepted May 10, 2014

Published October 27, 2014

DOI http://dx.doi.org/10.4238/2014.October.27.15

\begin{abstract}
This study aimed to determine the protective effect and mechanism of neutrophil gelatinase-associated lipocalin (NGAL) in rat kidney on ischemia/reperfusion injury (I/R). The rat I/R model was set up by cutting one kidney and clamping the contralateral renal pedicle for $45 \mathrm{~min}$. Male SD rats were randomly divided into sham-operation, $\mathrm{I} / \mathrm{R}$ and NGAL groups. Hematoxylin-eosin staining was performed to observe the renal pathological changes in the 3 groups; serum creatinine (Scr) and blood urea nitrogen (BUN) determined in blood samples taken from the inferior vena cava $24 \mathrm{~h}$ after the reperfusion were measured; TUNEL was used to observe the apoptosis of renal tubular epithelial cells; immunohistochemistry was performed to evaluate the expressions of Bax and activated caspase-3; Western blotting was used to determine the expression changes in apoptotic proteins Fas and Bcl2. Compared with the I/R group, Scr and BUN of the NGAL group were $63.400 \pm 11.908$ vs $121.857 \pm 17.151 \mu \mathrm{M}$ and $14.840 \pm 2.868$ vs 28.557 $\pm 6.434 \mathrm{mM}$, respectively. The number of apoptotic tubular epithelial cells was reduced $(7.800 \pm 1.924$ vs $15.400 \pm 3.049)$; the expression of
\end{abstract}


renal tissue Fas mRNA of the NGAL group was decreased $(2.34 \pm 0.51$ vs $6.84 \pm 2.34)$; the expression of the Bax protein was lower (7.440 \pm 1.640 vs $15.456 \pm 1.955 \%)$; the expression of the CC 3 protein was decreased $(3.171 \pm 0.321$ vs $7.291 \pm 1.059 \%)$, while the expression of the Bcl-2 protein increased $(6.91 \pm 1.64$ vs $5.30 \pm 1.48), \mathrm{P}<0.05$. NGAL had a protective effect towards the renal tubular epithelial cells in $\mathrm{I} / \mathrm{R}$, and the effect might have been associated with the reduction in apoptosis and the altered expression of apoptotic proteins, which would thereby reduce tissue damage and protect the kidney.

Key words: NGAL; Tubular epithelial cells; Ischemia-reperfusion; Apoptosis; Apoptotic protein

\section{INTRODUCTION}

Acute renal failure is a common critical illness, and although blood purification therapy has been widely popularized, its incidence and mortality still remain high. In recent years, acute renal injury (AKI) has accounted for $67.2 \%$ of the multi-organ injuries caused by global emergencies and natural disasters. To detect renal injury earlier and treat it in time, the international medical community has increasingly referred to the term AKI (El Sabbahy and Vaidya, 2011). The etiologies of AKI are various, with complex mechanisms, among which ischemia and toxins are the 2 main causes. It was reported that the incidence rate of AKI in all hospitalized patients is $5-35 \%$, and the mortality is increased 2- to 5-fold (Kanbay et al., 2010). Through gene analysis of the biological processes of ischemic AKI, it was found that neutrophil gelatinase-associated lipocalin (NGAL) was one of the gene products that were clearly expressed early after the renal ischemic injury (Zhao et al., 2010). Investigators have demonstrated that the administration of exogenous NGAL could significantly reduce the morphological and functional damages in murine renal ischemia-reperfusion (I/R), and reduce number of the apoptotic renal tubular cells (Mishra et al., 2004), where apoptosis has been considered to be the major form of death of renal tubular epithelial cells after renal ischemic injury (Dagher, 2004). Apoptosis includes the mitochondrium-mediated endogenous apoptosis pathway and the death receptor-mediated extrinsic apoptotic pathway (Brunelle and Letai, 2009). Gong et al. (2012) previously published that NGAL attenuated renal injury and inhibited renal tubular epithelial cell apoptosis via Bcl-2/Bax signaling pathways. According to the aforementioned reports, it was hypothesized that NGAL has a protective role for renal I/R injury, which may be correlated with reduced renal tubular epithelial cell apoptosis. To test this hypothesis, the effect of NGAL on renal function was studied by determining cleaved caspase-3 (CC3) and pro-apoptotic Bax protein in a rat ischemia/reperfusion model, aiming to understand the underlying mechanisms for clinical applications.

\section{MATERIAL AND METHODS}

\section{Animals and reagents}

This study was carried out in strict accordance with the recommendations in the Guide for the Care and Use of Laboratory Animals of the National Institutes of Health. The 
animal use protocol had been reviewed and approved by the Institutional Animal Care and Use Committee (IACUC) of the Shanghai Jiaotong University. Thirty male SD rats weighing 180-220 g were purchased from Shanghai Xipuer-Bikai Experimental Animal Co., Ltd.; LCN2/Lipocalin-2 recombinant rats (NAGL) were purchased from Beijing Sino-Biological Inc. Rabbit anti-mouse Fas monoclonal antibody (C18C12), rabbit anti-mouse Bcl-2 monoclonal antibody (50E3), and rabbit anti-rat polyclonal antibody (CC3) were purchased from Cell Signaling Technology Company (USA). Rabbit anti-rat Bax polyclonal antibody was purchased from Santa Cruz Biotechnology Company (USA); ready-to-use enzyme-labeled goat anti-rabbit secondary antibody was purchased from Fuzhou Maixin Company (China). TUNEL apoptosis detection kit and DAB staining kit were purchased from Wuhan Boster Co., Ltd. (China).

\section{Animal grouping, model preparation and the administration methods}

The 30 rats were randomly divided into the sham-operation group (SO group), I/R group and NGAL group, with $\mathrm{N}=10$ in each group. The rats were fasted $12 \mathrm{~h}$ before the operation, while having free access to water; after anesthetizing the rat by intraperitoneal injection of $1 \%$ sodium pentobarbital $(0.5 \mathrm{~mL} / 100 \mathrm{~g})$, an incision was made along the abdominal linea alba, and the left kidney was cut, freeing the right renal pedicle. The right renal pedicle was then occluded by minimally invasive arterial clamping. The kidney's rapid turning from rosy to purple under naked-eye observation indicated successful occlusion. After $45 \mathrm{~min}$, the clamp was released to perform the reperfusion, and the right kidney's turning from dark colored to rosy within 5 min meant the reperfusion was successful. The SO group only underwent the separation of the right renal pedicle but not the occlusion. NGAL, $50 \mu \mathrm{g}$, was preoperatively dissolved in $2 \mathrm{~mL}$ sterile distilled water and mixed thoroughly, and $0.5 \mathrm{~mL}$ solution was then injected via the tail $30 \mathrm{~min}$ before ischemia, at ischemia, and 30 and $60 \mathrm{~min}$ after ischemia vein; the same amount of saline was administered to the $\mathrm{SO}$ and $\mathrm{I} / \mathrm{R}$ groups.

\section{Specimen collection}

After $24 \mathrm{~h}$ of reperfusion, the animals were anesthetized by intraperitoneal injection of $1 \%$ sodium pentobarbital, followed by laparotomy along the original incision to expose the right kidney. A 3-mL blood sample was taken from the inferior vena cava, and the right kidney was then removed and fixed in 10\% neutral formalin. The collected blood samples were centrifuged at $40^{\circ} \mathrm{C}$ and $2500 \mathrm{rpm}$ for $15 \mathrm{~min}$, the upper serum layer was collected and stored at $-80^{\circ} \mathrm{C}$ for future evaluation of renal function.

\section{Determination of renal function}

Serum creatinine (Scr) and blood urea nitrogen (BUN) were determined with a Siemens Advia 2400 automatic biochemical analyzer.

\section{Renal pathological changes}

The specimens were fixed in $10 \%$ neutral formalin, and then embedded and sliced. The paraffin sections $(3 \mu \mathrm{m})$ were routinely dewaxed and rehydrated for hematoxylin staining 
for $10 \mathrm{~min}$. The slides were then rinsed with water, differentiated with $1 \%$ hydrochloric acid alcohol, stained with eosin for 2 min, dehydrated, cleared, and mounted. The renal pathological structure was then observed under a light microscope.

\section{Apoptosis of renal tubular epithelial cells by TUNEL assay}

The 3- $\mu \mathrm{m}$ kidney paraffin sections were analyzed according to TUNEL-staining kit instructions. The specimen slices of 3 rats in each group were selected, and 5 non-overlapping views at the cortico-medullary border of each slice were randomly selected with a powerful microscope $(400 \mathrm{X})$ to calculate the number of positive cells.

\section{Determination of Fas and Bcl-2 protein levels in tubular epithelial cells by Western blotting}

Rat kidney tissue was frozen in liquid nitrogen and total protein was extracted. The BCA Protein Assay kit was used for protein quantitation.

\section{Observation of Bax and activated CC3 by immunohistochemistry}

The paraffin sections were dewaxed, rehydrated, rinsed with distilled water, blocked with $3 \% \mathrm{H}_{2} \mathrm{O}_{2}$ in methanol, and washed with PBS at room temperature for $30 \mathrm{~min}$. Next, add sodium citrate to each section, experienced high fire for $2 \times 8 \mathrm{~min}$, cooled to room temperature again, followed by washing with PBS. The slides were incubated with primary antibody overnight at $40^{\circ} \mathrm{C}\left(\mathrm{CC} 3,1: 200\right.$; Bax, 1:50), and then at $37^{\circ} \mathrm{C}$ for $1 \mathrm{~h}$, followed by washing with PBS. The sections were incubated with the ready-to-use enzyme-labeled secondary antibody at $37^{\circ} \mathrm{C}$ for $30 \mathrm{~min}$, washed with PBS, controlled-DAB stained under the microscope, restained with hematoxylin for $8 \mathrm{~min}$, differentiated with $1 \%$ hydrochloric acid alcohol, bluereversed with distilled water, dehydrated, cleared, and mounted.

\section{Immunohistochemical image analysis}

An Axioplan 2 imaging microscope with image analysis system (provided by School of Medicine, Shanghai Jiaotong University) was used. Five fields (400X magnification) of each slice were randomly selected under the light microscope for the high-resolution picture. The images were then segmented and binarization performed. The measurement parameters were uniformly selected, automatically measured and analyzed for the positive expression rate of the protein.

\section{Statistical methods}

All data were subjected to statistical analysis with the SPSS13.0 statistical software. The data are reported as means \pm standard deviation. After testing for homogeneity of variance, comparison of multiple samples was performed using completely randomized design analysis of variance, and the comparison of two means was done using the LSD test. $\mathrm{P}<0.05$ indicated a significant difference. 


\section{RESULTS}

\section{Determination results of renal function tests}

At $24 \mathrm{~h}$ after reperfusion, Scr and BUN of the I/R group was significantly increased (P $<0.05)$ compared with the SO group, while these parameters were significantly lower in the NAGL group compared with the I/R group $(\mathrm{P}<0.05$; Table 1$)$.

Table 1. Serum creatinine and blood urea nitrogen and Bun values of the 3 groups.

\begin{tabular}{lcc}
\hline Group & Serum creatinine $(\mu \mathrm{M})$ & Blood urea nitrogen $(\mathrm{mM})$ \\
\hline SO & $24.000 \pm 3.829$ & $5.814 \pm 1.961$ \\
I/R & $121.857 \pm 17.151^{*}$ & $28.557 \pm 6.434^{*}$ \\
NAGL & $63.400 \pm 11.908^{* *}$ & $14.840 \pm 2.868^{* \#}$ \\
\hline
\end{tabular}

Data are reported as means $\pm \mathrm{SD} . * \mathrm{P}<0.05$ compared with the $\mathrm{SO}$ group, ${ }^{*} \mathrm{P}<0.05$ compared with the I/R group. $\mathrm{SO}=$ sham-operation; $\mathrm{I} / \mathrm{R}=$ ischemia/reperfusion injury; $\mathrm{NAGL}=$ neutrophil gelatinase-associated lipocalin.

\section{Renal pathological changes}

Under light microscopy, the tubular epithelial cells of the SO group exhibited no significant necrosis or degeneration, and the kidney structure was clear. On the other hand, the kidney structure of the I/R group clearly showed damage: interstitial edema, swelling and degeneration of tubular epithelial cells, nucleus absent, epithelial cells flatted, brush-like border lost, basement membrane bare, and formation of tubes. Compared with the I/R group, the tubular epithelial cell injury of the NGAL group was significantly less, where only a few cells exhibited vacuolar degeneration, with the brush-like border lost, while the shape of the renal tubule was well maintained (Figure 1).

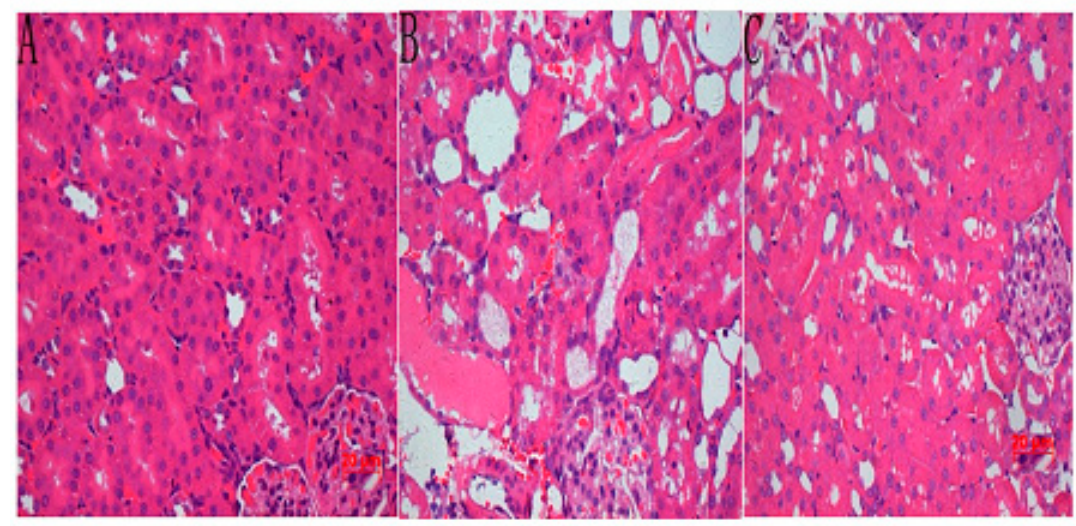

Figure 1. HE staining results of the 3 groups (HE, 400X). A. SO group. B. I/R group. C. NAGL group. For abbreviations, see legend to Table 1.

\section{Apoptosis of tubular epithelial cells}

The TUNEL-positive cells were mainly observed among the renal tubular epithelial cells, while rare in the glomerulus and stroma. At $24 \mathrm{~h}$ after I/R, the number of apoptotic 
epithelial cells increased, while the extent of apoptosis in the NAGL group was significantly decreased $(\mathrm{P}<0.05)$, and the apoptosis occurred rarely in the SO group (Table 2 and Figure 2$)$.

Table 2. Comparison of apoptotic cells of the 3 groups.

\begin{tabular}{lccc}
\hline & SO group & I/R group & NGAL group \\
\hline Apoptotic cells (N/field) & $1.800 \pm 1.140$ & $15.400 \pm 3.049^{*}$ & $7.800 \pm 1.924 * \#$ \\
\hline
\end{tabular}

Data are reported as means $\pm \mathrm{SD}$ for $\mathrm{N}=10$ in each group. ${ }^{*} \mathrm{P}<0.05$ compared with the $\mathrm{SO}$ group; ${ }^{*} \mathrm{P}<0.05$ compared with the I/R group. For abbreviations, see legend to Table 1.
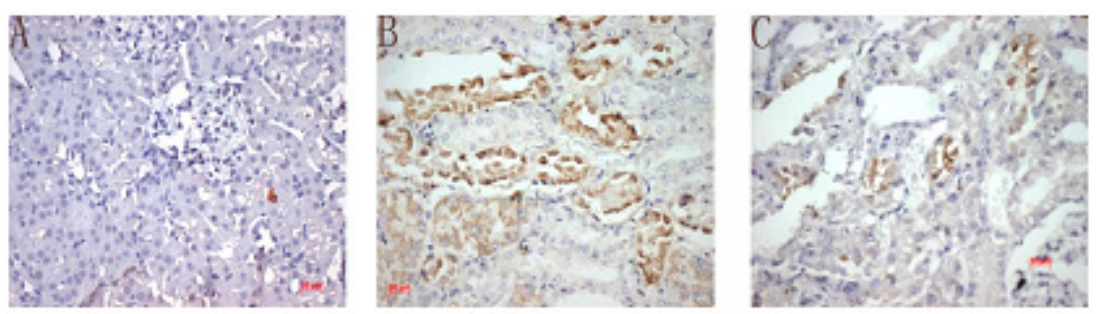

Figure 2. Apoptotic results with TUNEL staining (HE, 400X). A. SO group. B. I/R group. C. NAGL group. For abbreviations, see legend to Table 1 .

\section{Western blot results}

Compared with the I/R group, the expression of renal tubular Fas in the NGAL group was decreased, while the expression of the Bcl-2 protein was increased (Table 3 ).

Table 3. Relative mRNA contents of Fas and Bcl-2 of the 3 groups.

\begin{tabular}{lccc}
\hline Gene & SO group & I/R group & NGAL group \\
\hline Fas & 1 & $6.84 \pm 2.34$ & $2.34 \pm 0.51^{*}$ \\
Bcl-2 & 1 & $5.30 \pm 1.48$ & $6.91 \pm 1.64^{* *}$ \\
\hline
\end{tabular}

Data are reported as means $\pm \mathrm{SD}$ for $\mathrm{N}=5$ in each group. $* \mathrm{P}<0.01$ compared with the $\mathrm{SO}$ group. $* * \mathrm{P}<0.05$ compared with the I/R group. For abbreviations, see legend to Table 1.

\section{Positive expression of $\mathrm{CC} 3$ and Bax}

Activated caspase-3 is mainly localized in renal tubular epithelial cells, and has no expression in the renal glomerulus. Positive expression of the $\mathrm{I} / \mathrm{R}$ group was significantly higher than that of the SO group, while that of the NAGL group was significantly lower compared with the $\mathrm{I} / \mathrm{R}$ group $(\mathrm{P}<0.05)$. Bax was mainly expressed in renal tubular epithelial cells, with little expression in the group, and the expression of Bax after $\mathrm{I} / \mathrm{R}$ significantly increased, while that in the NAGL group was lower, with the difference being statistically significant $(\mathrm{P}<0.05$; Table 4$)$.

\begin{tabular}{lcc} 
Table 4. Positive expression rate of CC3 and Bax protein of the 3 groups. \\
\hline Group & CC3 (\%) & Bax (\%) \\
\hline SO & $0.608 \pm 0.148$ & $3.716 \pm 0.482$ \\
I/R & $7.291 \pm 1.059^{*}$ & $15.456 \pm 1.955^{*}$ \\
NAGL & $3.171 \pm 0.321^{* *}$ & $7.440 \pm 1.640^{* *}$ \\
\hline Data
\end{tabular}

Data are reported as means $\pm \mathrm{SD} . * \mathrm{P}<0.01$ compared with the $\mathrm{SO}$ group. $* * \mathrm{P}<0.05$ compared with the I/R group. For abbreviations, see legend to Table 1. 


\section{DISCUSSION}

The kidney is an organ with high perfusion, and sensitive to I/R injury. Renal I/R injury mainly appears in renal tubule and stroma, indicating the swelling, necrosis and shedding of the renal tubular epithelial cells, and the expansion of renal tubular lumen, where protein and red blood cells can be seen in the lumen along with the infiltration of interstitial inflammatory cells. In this study, HE staining revealed evident necrosis, shedding and edema in I/R renal tubular epithelial cells. Although the tubular epithelial cells still showed swelling, necrosis was reduced in the NGAL group, and the degree of injury abated. Mori et al. (2005) reported that in the early stage of severe renal failure in the mouse I/R model, a single dose of NGAL $(10 \mu \mathrm{g})$ afforded significant renal protection and reduced azotemia.

NGAL is also known as lipid-transport protein 2 (lipocalin-2) and $24 \mathrm{p} 3$, one member of the lipocalin superfamily. It was initially found in activated neutrophils, and found in other types of cells, including the renal tubular cells after a variety of injuries (Zhao et al., 2010). Mishra et al. (2004) compared the protective effects of exogenous NGAL on the kidneys, which was administered $1 \mathrm{~h}$ before ischemia, at ischemia and $1 \mathrm{~h}$ after ischemia. The results revealed that NGAL could have a protective effect at the above 3 time points, indicating that NGAL could be effective within $1 \mathrm{~h}$ after ischemia. In the present study, the exogenous rat recombinant NGAL protein was intravenously injected through the tail vein 30 min before ischemia, at ischemia, and 30 and $60 \mathrm{~min}$ after ischemia, aiming to determine its effect and mechanism on renal tubular epithelial cells. The results showed that exogenous NGAL significantly inhibited the increase in Scr and BUN in I/R rats, and although the renal tubular epithelial cells still showed swelling, necrosis was reduced and the degree of injury also reduced.

When apoptosis occurs, broken DNA fragments appear in nucleus, and the TUNEL method can detect these fragments with high sensitivity. In this experiment, the TUNEL assay was applied in a morphological way. Extensive apoptosis of renal tubular epithelial cells occurred in I/R rats, while after the administration of the NGAL protein through the tail vein, the number of apoptotic cells was reduced, indicating the protection afforded by NGAL may be related to the reduction in apoptosis. In recent years, investigators also found that the NGAL-overexpressing renal tubular epithelial cells after $\mathrm{I} / \mathrm{R}$ were negative in TUNEL staining, and that the renal tubular epithelial cells that expressed NGAL and PCNA (proliferating cell nuclear antigen) showed evident proliferation and regeneration (Mishra et al., 2003), which seemed to explain the close relationship of the NGAL protection and the apoptosis reduction from another angle. The results of this study showed that the apoptosis mainly occurred among the tubular epithelial cells at the cortico-medullary border, which matched the report by Sutton (2009) that the cortico-medullary border was the most serious region of tubular injury (Chipuk et al., 2010). The apoptosis in the NGAL group was significantly less than in the I/R group, suggesting that NGAL could play a role in kidney protection by inhibiting apoptosis.

At the core of apoptosis are the cysteine-containing aspartate-specific proteases (caspases). When caspase is activated, the key enzymes and structure proteins inside the cells are degraded, causing cell death. There are two main ways. 1) Caspases regulate mitochondrialmediated apoptosis, and Bcl-2 family plays the decisive role in this way, where the Bcl-2/Bax system is the endogenous signal pathway, which would regulate apoptosis, where Bcl-2 can antagonize apoptosis while Bax plays the opposite role. When receiving the signal, Bax would be transferred from the cytoplasm to the outer mitochondrial membrane, promoting the formation of an apoptosome, which contains caspase-9 pro-enzyme, thus starting the cascade reactions of 
caspase activation events. 2) Fas is a type I membrane protein, and FasL is a type II membrane protein, belonging to the protein superfamily of tumor necrosis factor. FasL is the death factor, and Fas is its receptor. The activation of Fas induced by FasL would result in the aggregation and activation of the inactive Fas compound, forming the death-inducing signal complex (FasDISC), which would shear the activated caspase- 8 and activate caspase- 3 , leading to the apoptosis of Fas-expressing cells (Krauss, 2005). In the present study, Western blot analysis was adopted for determining the expression levels of the Fas and Bcl-2 genes in the apoptotic signal transduction pathway. Compared with I/R group, the expression of Fas mRNA in renal tubular epithelial cells of the NGAL group declined, while the expression of Bcl-2 mRNA increased, showing significant differences, indicating that NGAL may have renoprotective activity by reducing the expression of Fas and enhancing the expression of Bcl-2, thereby inhibiting the occurrence of apoptosis. In vitro experiments also proved that NGAL could inhibit the apoptosis of HK-2 cells subjected to hypoxia and reoxygenation (Cui et al., 2011).

The reason for this anti-apoptotic effect may be that NGAL can combine with siderophore and iron to form a complex and then release intracellular iron via the endocytosis of giantin receptors of tubular epithelial cells (megalin) and NGAL receptors (Schmidt-Ott et al., 2006). On the one hand, when iron enters the target cells, it will reduce the generation of the extracellular ROS, thereby reducing oxidative stress and cell damage. ROS not only can directly damage DNA and induce apoptosis or attack proteins to induce apoptosis, but may also act on the cell membrane and induce lipid peroxidation, thus affecting the cell signal transduction system to induce apoptosis. On the other hand, NGAL could release iron inside the cells, which is necessary to maintain the stable spatial conformation of many active proteins; it could act as a unique-regulating factor to activate gene transcription and the transcriptional mechanism, thus improving the expression of iron-dependent genes (Yang et al., 2002). Therefore, the gain of intracellular iron is very important for the regulation of cell proliferation, apoptosis and differentiation. In human adenocarcinoma cells, when the iron is lost because of the chelation, cell proliferation can be inhibited and the apoptosis induced (Sun et al., 2009). Alcantara et al. (2001) also confirmed that the intracellular iron reduction caused by antagonizing transferrin could lead to cell cycle arrest and the inhibition of cyclin, bcl-2 and myc (a protein encoded by a proto-oncogene) family.

This study also used an immunohistochemical method to observe the Bax changes during the experiment, and found that Bax mainly existed in the cytoplasm of renal tubular epithelial cells, and that there was only a small amount of expression in the SO group, which was consistent with the literature (Tourneur and Chiocchia, 2010). When I/R occurred, cytosolic Bax increased rapidly, while after administration of exogenous NGAL, the expression of Bax was significantly reduced, suggesting that Bax was involved in renal I/R, and that the exogenous NGAL could inhibit the expression of Bax. Caspases belong to a protease family that plays an important role in the regulation of apoptosis. Caspase- 3 is the primary executioner of the family, where is the downstream molecule, existing in the cytoplasm in the zymogen form under normal circumstances. When subjected to a variety of extracellular stimuli, the main upstream initiators, caspase- 8 and caspase- 9 , would be enzyme-cleaved and activated and enter the nucleus, and activated CC 3 would hydrolyze many downstream target proteins and cause apoptosis (Takemoto et al., 2003; Pop and Salvesen, 2009). In the present study, it was also found that activated CC3 mainly existed in the nucleus of the tubular epithelial cell, while there was only a very small amount expressed in the SO group. When I/R occurred, the expression of $\mathrm{CC} 3$ was significantly increased compared with the SO group, which was concordant with ex- 
perimental results of Raff et al. (2005). After the administration of exogenous NGAL, expression was significantly reduced. Therefore, NGAL may have renoprotective activity by reducing the expression of Bax and inhibiting the activation of caspase-3, thereby inhibiting apoptosis.

In summary, this study revealed that NGAL could effectively reduce the number of apoptotic tubular epithelial cells, achieving a renoprotective effect by inhibiting the activation of caspase- 3 and lowering the expressions of Bax and Fas, while enhancing the expression of Bcl-2, thereby inhibiting apoptosis. The relationship between NGAL and AKI was elaborated, providing a theoretical basis for clinical AKI treatment.

\section{ACKNOWLEDGMENTS}

Research supported by Scientific Research Project of Shanghai Health Bureau (\#20114331) and the Advanced Medical Cooperation Project of Songjiang Health Bureau of Shanghai (2011).

\section{REFERENCES}

Alcantara O, Kalidas M, Baltathakis I and Boldt DH (2001). Expression of multiple genes regulating cell cycle and apoptosis in differentiating hematopoietic cells is dependent on iron. Exp. Hematol. 29: 1060-1069.

Brunelle JK and Letai A (2009). Control of mitochondrial apoptosis by the Bcl-2 family. J. Cell Sci. 122: 437-441.

Chipuk JE, Moldoveanu T, Llambi F, Parsons MJ, et al. (2010). The BCL-2 family reunion. Mol. Cell 37: 299-310.

Cui LY, Yang S and Zhang J (2011). Protective effects of neutrophil gelatinase-associated lipocalin on hypoxia/ reoxygenation injury of HK-2 cells. Transplant. Proc. 43: 3622-3627.

Dagher PC (2004). Apoptosis in ischemic renal injury: roles of GTP depletion and p53. Kidney Int. 66: 506-509.

El Sabbahy M and Vaidya VS (2011). Ischemic kidney injury and mechanisms of tissue repair. Wiley Interdiscip. Rev. Syst. Biol. Med. 3: 606-618.

Gong L, Yu H, ZhuGe Y and Yu Q (2012). Neutrophil gelatinase-associated lipocalin protects renal tubular epithelial cell in ischemic/reperfusion injury rats via apoptosis-regulating proteins. Ren. Fail. 34: 777-783.

Kanbay M, Kasapoglu B and Perazella MA (2010). Acute tubular necrosis and pre-renal acute kidney injury: utility of urine microscopy in their evaluation - a systematic review. Int. Urol. Nephrol. 42: 425-433.

Krauss G (2005) Intracellular Signal Transduction: The Protein Cascades of the MAP Kinase Pathways. In: Biochemistry of Signal Transdution and Regulation. Wiley-VCH Verlag GmbH \& Co. KGaA, Weinheim, 389-392.

Mishra J, Ma Q, Prada A, Mitsnefes M, et al. (2003). Identification of neutrophil gelatinase-associated lipocalin as a novel early urinary biomarker for ischemic renal injury. J. Am. Soc. Nephrol. 14: 2534-2543.

Mishra J, Mori K, Ma Q, Kelly C, et al. (2004). Amelioration of ischemic acute renal injury by neutrophil gelatinaseassociated lipocalin. J. Am. Soc. Nephrol. 15: 3073-3082.

Mori K, Lee HT, Rapoport D, Drexler IR, et al. (2005). Endocytic delivery of lipocalin-siderophore-iron complex rescues the kidney from ischemia-reperfusion injury. J. Clin. Invest. 115: 610-621.

Pop C and Salvesen GS (2009). Human caspases: activation, specificity, and regulation. J. Biol. Chem. 284: 21777-21781.

Raff U, Schneider R, Gambaryan S, Seibold S, et al. (2005). L-Arginine does not affect renal morphology and cell survival in ischemic acute renal failure in rats. Nephron Physiol. 101: 39-50.

Schmidt-Ott KM, Mori K, Kalandadze A, Li JY, et al. (2006). Neutrophil gelatinase-associated lipocalin-mediated iron traffic in kidney epithelia. Curr. Opin. Nephrol. Hypertens. 15: 442-449.

Sun X, Ge R, Cai Z, Sun H, et al. (2009). Iron depletion decreases proliferation and induces apoptosis in a human colonic adenocarcinoma cell line, Caco2. J. Inorg. Biochem. 103: 1074-1081.

Sutton TA (2009). Alteration of microvascular permeability in acute kidney injury. Microvasc. Res. 77: 4-7.

Takemoto K, Nagai T, Miyawaki A and Miura M (2003). Spatio-temporal activation of caspase revealed by indicator that is insensitive to environmental effects. J. Cell Biol. 160: 235-243.

Tourneur L and Chiocchia G (2010). FADD: a regulator of life and death. Trends Immunol. 31: 260-269.

Yang J, Goetz D, Li JY, Wang W, et al. (2002). An iron delivery pathway mediated by a lipocalin. Mol. Cell 10: 1045-1056.

Zhao C, Ozaeta P, Fishpaugh J, Rupprecht K, et al. (2010). Structural characterization of glycoprotein NGAL, an early predictive biomarker for acute kidney injury. Carbohydr. Res. 345: 2252-2261. 